\title{
Design and Production of Educational VR Contents Learning about Design's Principle and Flow
}

\author{
Bongkwan Moon1), Sangin Nam²), Dongkyum $\mathrm{Kim}^{2}$, Hyeonjeong $\mathrm{Kim}^{2}$, Eunju Park ${ }^{3}$ ) and \\ Hankyu Lim $\left.^{4}\right)$
}

\begin{abstract}
In this paper, the virtual reality (VR) technology, which fused and implemented information technologies, has become reality as many consumer VR devices are launched in the market and users employ them more than ever. This study designs and implements exhibition space VR for education purpose where users employ VR devices and select the design principle and flow to learn the contents. The contents designed and implemented in this study improved a sense of immersion by producing unorganized and fragmentary design shape principles as a form of VR contents. Thus, this study expects users to understand and be interested in the design principles more easily.
\end{abstract}

Keywords: VR, Contents, Design, 3D Max, Unity, HoloRens

\section{Introduction}

VR contents refer to simulation contents by applying VR technology for users to experience virtual reality indirectly[1]. VR technology accompanied by hardware advance now enables implementation of virtual world, which was not imaginable in the past. With excellent performance, realistic expression, VR can now express a variety of realistic worlds[2]. Thus, as VR technology that create a virtual space has advanced using virtual images consisting of background and objects as a tool to share and expand human experiences, users can now indirectly experience various fields. The development and commercialization of VR systems are increasing more recently[3]. The VR industry has been expanded into various ranges including

Received(September 11, 2019), Review Result(1st: October 07, 2019, 2nd: November 13, 2019), Accepted(December 23, 2019)

1) (Student) 36729 Dept of Multimedia Engineering, Andong National University, Andong, Korea email: mo1359on@naver.com

2) (Student) 36729 Dept of Multimedia Engineering, Andong National University, Andong, Korea email: nsi8953@naver.com, fwpd@naver.com, hjung5034@naver.com

3) (Professor) 36729 Dept of Multimedia Engineering, Andong National University, Andong, Korea email: eunju@anu.ac.kr

4) (Professor, Corresponding Author) 36729 Dept of Multimedia Engineering, Andong National University, Andong, Korea

email: hklim@anu.ac.kr 
entertainment such as games and videos, aviation, medicine, military training etc., and even VR cafeterias have emerged as one of the playgrounds for the youth[4]. VR improves attention to surroundings and motivation through immersion and spatial presence in a specific environment as it is characterized with increasing learner's interests on learning by providing life-like experiences in a virtual space[5].

Although the key virtue of design is creativity, imitation is also closely related to creativity at the same time, which is a required component in defining a design problem and finding a solution[6]. Creators attempt to creatively improve existing designs by following or imitating existing designs, and designs themselves are also developed through learning. Thus, good designs may not be possible without knowing the design flows and techniques as there are shapes and principles that become prototypes of design learning. For example, the perspective principle of Gestalt psychology and Lubin's visual illusion, color layout and combination, and color symbolization are some of the important factors in design work, and design variability can be expanded only when visual-perceptual constancy is maintained[7]. However, these design principles are normally unorganized and divided in a fragmented manner. Thus, this study aims to make a learning space where design principles and flows can be learned by developing a good immersive VR content for users to understand the design principles and flows easily as well as attracting interests. It also helps users to learn the design principles and flows as if they freely watch the works in an exhibition by providing a learning space through VR contents so that participants can learn without time and space limitation. The "VR content for learning design principle and flow" in this study consists of three categories: photo, principle, and font, each of which is also available through A4-size catalog to be easily referenced in daily living. This study produced required space modeling using three dimensional (3D) MAX, and a lively exhibition space was produced by designing a VR space in link with Unity.

This paper is organized as follows. Section 2 present related research in which equipment, program, and surveys required to develop the VR contents in this study are described. Section 3 describes the Strengths, Weaknesses, Opportunities, and Threats (SWOT) analysis, structural and user interface (UI) designs, and implementations. Section 4 present the conclusions along with the limitations and future research.

\section{Related Research}

\subsection{HoloLens}


Microsoft HoloLens is a wearable device based on mixed reality (MR) developed by Microsoft[8]. Although HoloLens, which employed the Windows holographic technology, uses virtual reality that displays complete virtual scenes, it is called MR that can display scanned 3D images of actual entities in the real screen and manipulate the scanned images freely, which is in contrast with augmented reality[9]. In addition, it is not a display headset that is connected to other devices such as personal computer (PC) or smartphone, but complete Windows PC functions are embedded in it.

This study produced a learning space via VR contents to utilize HoloLens, and aimed to have the same effect as if users watched works in exhibition or museum. The user participation is inducted through hand gesture, and users can experience data that show visual design principles and flows selectively.

\subsection{Unity and 3D Max}

Unity is a game engine that provides an environment to develop $3 \mathrm{D}$ and $2 \mathrm{D}$ video games, and an integrated authoring tool used to create interactive content authoring such as animation, construction visualization, and VR[10]. This study added many scenes using freely available Unity Personal. The connection to scenes was made to be used in HoloLens that recognized user's hand gesture by employing a click event.

3D MAX is a program to make natural and realistic 3D graphics through powerful 3D modeling, mapping, and rendering, which is widely used in visual effect, character animation, and games as it can be easily accessible and obtain good outcomes compared to other programs[11].

This study employed 3D MAX to model a frame that contains photos and exhibition space, and performed texture mapping.

\subsection{Survey}

[Fig. 1] shows the significant results in the survey, which was conducted to know the need of the authoring of VR content for learning design principle and flow designed and implemented in this study. The survey was conducted for online design community people from March 30 to April 3 in 2019.

The survey results exhibited that $11 \%$ of the respondents said they knew well about the design flow and trend whereas $59 \%$ of them said they did not know well about the design 
flow and trend. The survey results also showed that $64 \%$ of the respondents identified the design flow and trend through Internet media but few of them through books, experts, or advertisements.

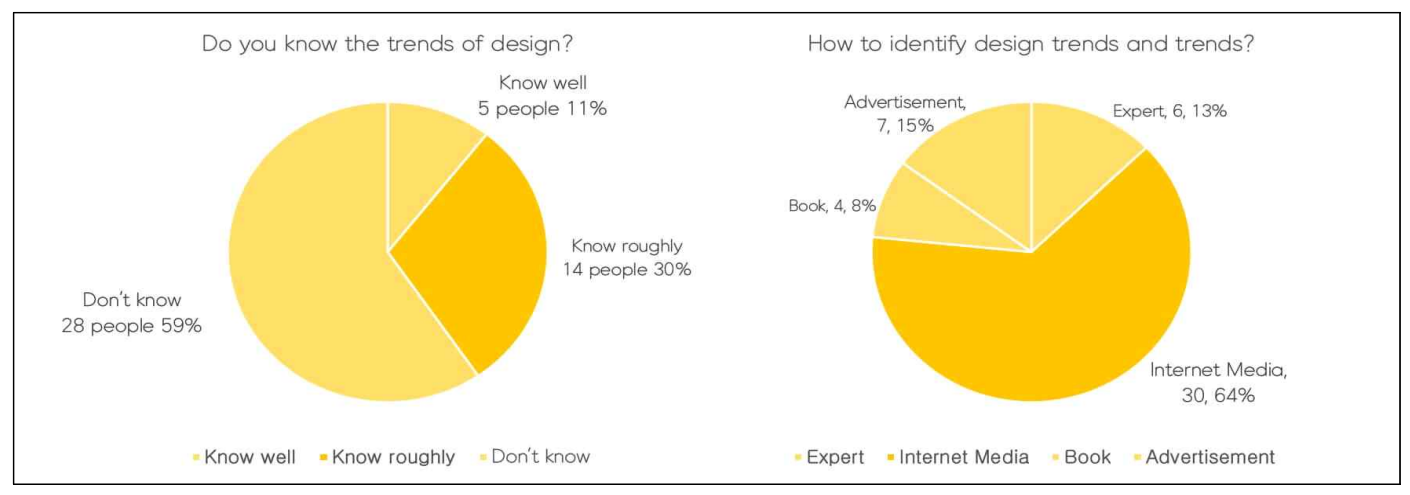

[Fig. 1] Survey

The above survey results summarized that many people did not know about the design flow, and methods to identify the design trend or information were very limited. Thus, the design learning space content using VR designed and authored in this study is one of the information supply methods for users to know the design principles and flows and learn about the design principles, and it is necessary to author such tool because it can play a role to facilitate learning more conveniently.

\section{Design and Implementation}

\subsection{SWOT Analysis}

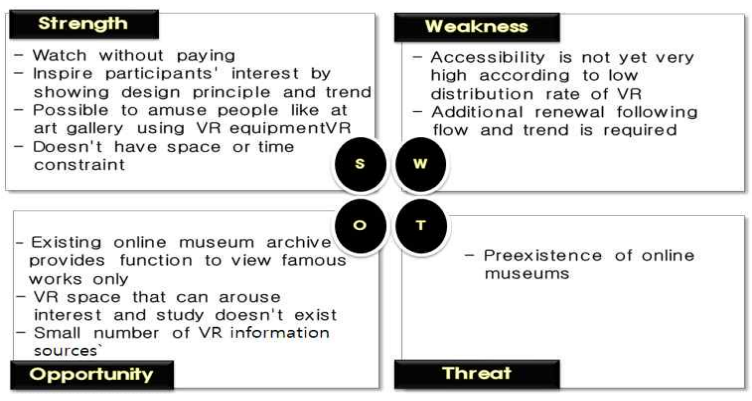

[Fig. 2] SWOT Analysis

The strengths of the "VR content for learning design principle and flow" are no limitation of 
time and space, no cost occurred, and induction of participant interest. The weaknesses are low accessibility due to unfamiliar concept of VR to users, and additional update is needed when contents are needed to be added. The opportunity is that there are not many elements that combine VR and learning in the market. The threat is that there are existing museum archives already. Thus, WO strategy that improves weaknesses into opportunities was adopted, aiming to pre-occupy not many VR learning spaces.

\subsection{Structural Design and UI Design}

[Fig. 3] shows the flow chart about the overall flow to store each of the works that belong to one of "Principle", "Photo", and "Font", which are the functions of the VR content authored in this study, and provide it to users. The flow marked by orange color refers to users going back to the previous screen by using the button.

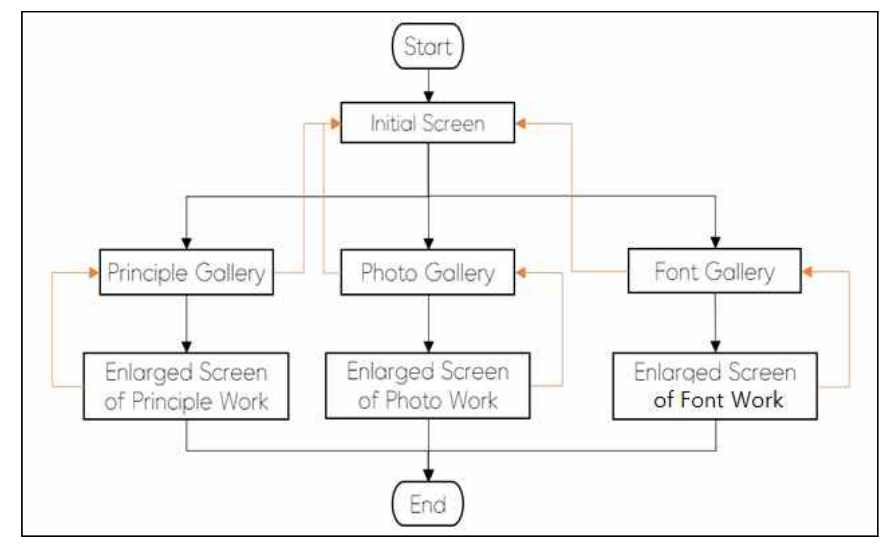

[Fig. 3] Flowchart

The "Photo" category introduces specific photographic composition and techniques suitable for each different circumstance along with the description of redesign. The "Principle" category describes the explanation and rationales attached to example images of the perspective principle. The "Font" category describes image authoring and explanation using famous fonts in each era.

[Fig. 4] shows the UI design of the first screen when executing the program by Unity. A user can select and move to the workspace where he/she would like to see. The topics of the work space belong to principle, photo, and font, respectively.

[Fig. 5] shows the UI design by which overall composition of work space regarding 
principle, photo, and font can be checked. A user can move to the start screen again by clicking the button or enlarging each of the works by clicking the preferred work in the screen.

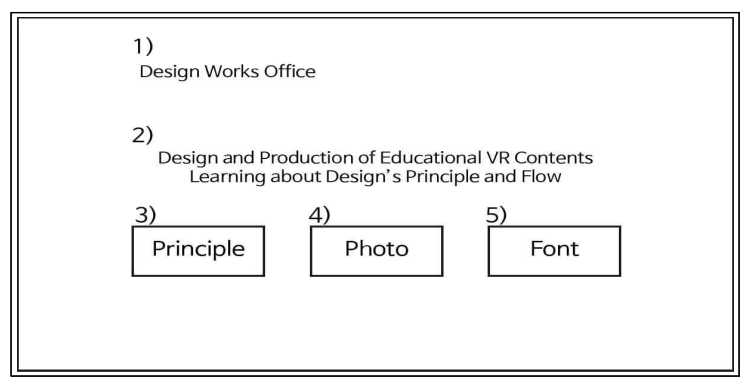

[Fig. 4] UI Design of Initial Screen

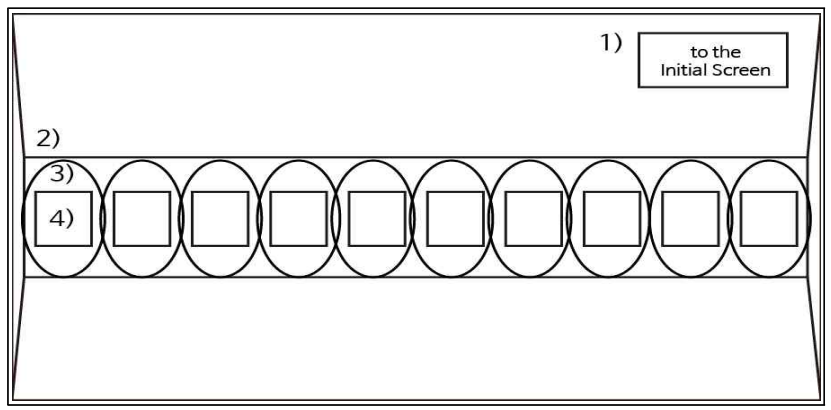

[Fig. 5] UI Design of Work Gallery Screen

\subsection{Implementation}

This study authored a work exhibition space using 3Ds MAX first, and then authored VR space using Unity to see the works. The application was built to be run in HoloLens, which can check the results as well. Each of the works in the application was also developed as a form of catalog, which can be seen even users are offline.

[Fig. 6] shows the screen implemented by Unity when users see the exhibition space where the works are displayed. In this space, a user can click his/her preferred picture arbitrarily and move the picture to a space where the work is enlarged, and the user appreciates the work.

[Fig. 7] shows the implemented screen that arranges the user-selected work to fit in the screen composition and enlarges the work to a user-preferred size. A button is made for users to return to the previous space where the works are displayed after seeing the work. When 
this button is clicked, a user can move back to the space where the works are listed.

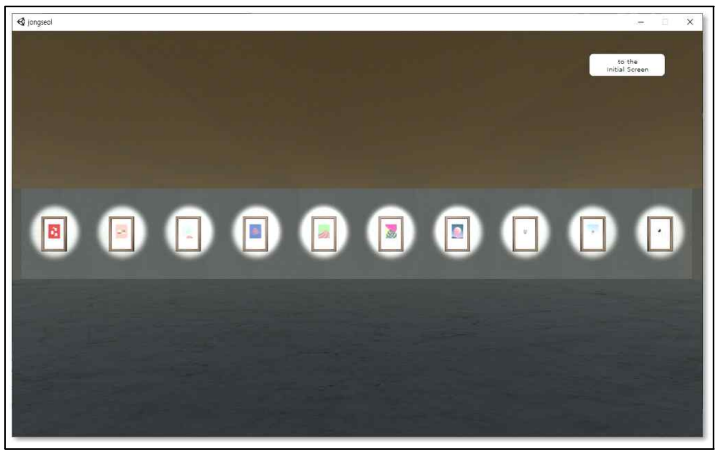

[Fig. 6] Implementation Screen of Work Gallery

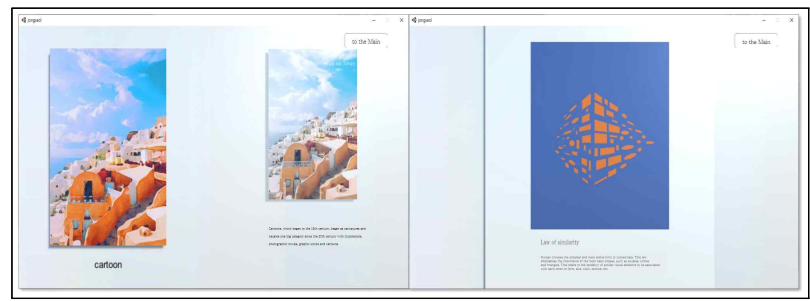

[Fig. 7] Screen that magnifies the work of user's choice

[Fig. 8] shows the mock-up pictures to check the works as a form of catalog even on offline in addition to seeing the works through the program.

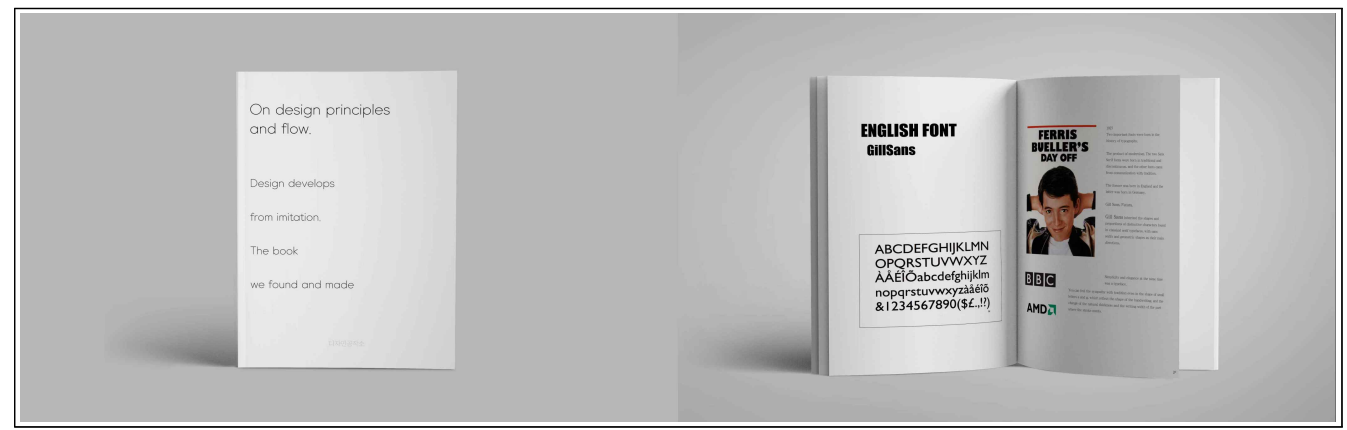

[Fig. 8] Woodcut Cover and Font Page

\section{Conclusion}


This study designed the learning space for design principles and flow and implemented the space with VR contents. Design work can be progressed by learning and there are principles about the prototype of design learning. Thus, this study designed a design learning space by which users can learn the design principles easily, and implemented the space using VR contents to increase the immersion as if users were placed in the design learning space. Three categories were made for users to learn the design principles easily, and a lively exhibition space was developed by linking the space modeling using 3D MAX with Unity. To make users understand the design principles easily, it provided images and texts, and works were available not only in online environments but also in mock-up form.

The market size has grown in a sense that exhibitions or galleries, which were regarded only one-off event before, can now be accessed to customers continuously by transcending the boundaries of space and time through using VR technology[12]. This study expects to help users to learn about the understanding and utilization of designs without limitations of time and space when participants learn. The contents authored in this study have a limitation: although this study aimed to produce more lively and more realistic atmosphere by matching the user's viewpoint with that of camera by utilizing 3D space during content authoring, this study did not apply this to the authored contents this time. To overcome this limitation, authoring space will be made with $3 \mathrm{D}$ rather than $2 \mathrm{D}$ to complement the project for future study.

\section{Acknowledgement}

This work was supported by a Research Grant(year 2019 year 2020) of Andong National University.

\section{References}

[1] YoungSoo Lee, A Study about Storytelling of Film Advertisement Contents Using VR Platform, Cartoon \& Animation Studies, (2018), No.53, pp.357-387, DOI: 10.7230/KOSCAS.2018.53.357

[2] JeongHyeon Han, Virtual Reality-based System Integration and Utilization, Korea University, Master's thesis, (2017)

[3] HeonJoo Lee, HwaSook Kim, Virtual - Augmented Reality Technology and Industrial Semiconductors, Weekly Technology Trend, (2018)

[4] https://blog.naver.com/shin_022/221188299313, Jan 1 (2018) 
[5] GaYoung Seol, A Study of Functional Connectivity and Structural Change of Brain in VR Content Based Life Sciences Learning, Korea National University of Education, Master's thesis, (2019)

[6] DongHa Kim, Imitation and Design, Archives of Design Research(ADR), (2013), Vol.26, No.2, pp.85-106.

[7] SeoJin Lee, A Study on Constancy of Flexible Identity from Gestalt Principles, A Journal of Brand Design Association of Korea, (2016), Vol.14, No.1, pp.183-194, DOI : 10.18852/bdak.2016.14.1.183

[8] Jun Pyo Lee, Development and Trends of Virtual, Augmented and Mixed Reality Technologies, Weekly ICT TrendS, (2019), No.1881, pp.2-15.

[9] http://blog.naver.com/PostView.nhn?blogId=tychung1\&logNo=220951994168, Mar 7 (2017)

[10] MyeonJae Lee, A Study on Game Production Education through Recent Trend Analysis of 3D Game Engine, Journal of the Korean Convergence Society, (2013), Vol.4, No.1, pp.15-20, DOI: 10.15207/JKCS.2013.4.1.015

[11] SeHun Kim, A Study on Functional Characteristic of 3D Pre-visualization Software - Focusing on the analysis of storyboard-dedicated software and 3D high-end software -, journal of the moving image technology associon of korea, (2012), Vol.1, No.17, pp.159-178, DOI: http://dx.doi.org/10.34269/mitak.2012.1.17.009

[12] http://www.etnews.com/20181122000175, Nov 22 (2018) 